\title{
Heat transfer in separated and reattached flow regions over a circular cylinder
}

\author{
by C. Meola, G. Cardone, G. M. Carlomagno and L. Marino
}

DETEC Università degli Studi di Napoli "Federico II", P.le Tecchio 80, 80125 Napoli, ITALY

\begin{abstract}
The behaviour of a circular cylinder longitudinally oriented to a uniform freestream is analysed by means of infrared thermography applied to the heated-thin-foil technique. Two different configurations of the cylinder leading edge are considered : a sharp and a round ones. Tests are carried out, in an open circuit wind tunnel with low turbulence intensity, for varying the velocity of the freestream and the angle of attack of the cylinder axis with respect to the oncoming flow. The experimental data are reduced in dimensionless form in terms of the Nusselt number $\mathrm{Nu}$; the position of the maximum $\mathrm{Nu}$, which is linked to the flow reattachment, depends strongly on the leading edge configuration and on the angle of attack.
\end{abstract}

\section{Introduction}

The problem of separation and reattachment of flow can be encountered in many engineering applications; in particular, the separation of flow at leading edges deserves attention because it may influence the whole flow regime over a surface. In general, flow separation occurs under adverse pressure gradient and with viscosity (laminar or turbulent) effects and is caused not only by a gradual process (as in the case of flow over a smooth surface) but also by a severe discontinuity of the tangent to the surface [1].

The flow field around a cylindrical body with cross-section placed in a longitudinal flow is characterized by different flow separation and reattachment according to the geometry of the cylinder leading edge and to the angle of attack of its axis with respect to the incoming flow. Ota [2], from experiments carried out on a sharp-edged circular cylinder immersed in a stream at zero angle of attack for Reynolds number $R e$ (based on the cylinder diameter) ranging from 24,900 to 53,600 , finds that the reattachment point of the bubble originating at the front sharp leading edge occurs at 1.6 diameters from the latter $(x / D=1.6)$. However, in a subsequent paper concerning heat transfer measurements, Ota and Kon [3] argue that the flow reattachment occurs at the position where a maximum is attained for the heat transfer coefficient, i.e. at $x / D=1.3$. For similar Reynolds number values, the same position for the heat transfer maximum is found by Sparrow et al. [4], who however demonstrate that the real flow reattachment occurs further downstream, and in particular at $x / D=1.6$. It should be mentioned that the free-stream turbulence intensity is $0.4-0.5 \%$ in the tests of Sparrow et al., while it is approximately $0.8 \%$ in the tests of Ota. Mean and fluctuating flow field measurements through hot wire anemometry in air are presented by Kiya et al. [5] on a sharp-edged circular cylinder for $R e=200,000, \alpha=0^{\circ}$ and with a free-stream turbulence intensity of about $0.2 \%$; the reattachment point is derived from hot wire measurements in the vicinity of the wall, as the location where the reverse-flow time fraction is equal to 0.5 , and is also found to occur at $x / D=1.6$. Carlomagno [6], from measurements of heat transfer coefficients in a low-turbulence $(\sim 0.09 \%)$ stream, for $R e$ ranging from 16,300 to 32,100 , finds a maximum heat transfer occurring at $x / D$ from 1.8 to 2.0 respectively, i.e. at a much higher value than those presented by previous researchers.

For a circular cylinder with a hemispherical nose, the evolution of the surface skin-friction lines with increasing incidence, at a Mach number equal to 1.2, is described by Peake and Tobak [7]. In the case of axisymmetric flow $\left(\alpha=0^{\circ}\right)$, a separation bubble still exists immediately downstream of the junction between the hemispherical nose and the cylindrical body; however, this bubble is 
much smaller than the one in the sharp-edged case and the reattachment point seems to be located at $x / D \cong 0.15$. As the angle of attack increases, the bubble first becomes non-symmetrical, and then tends to disappear from the windward side of the cylindrical part, so that for $\alpha>5^{\circ}$ a complex configuration of separation and reattachment lines develops, with the appearance in the flow of longitudinal concentrated vortices, which persists up to very high values of $\alpha$.

Measurements, carried out by Cardone et al. [8] in a closed-return Göttingen type wind tunnel (open test section) with turbulence intensity $0.9 \%$, and Reynolds number ranging from 84,700 up to 159,000 , show a maximum heat transfer at $x / D=1.2$ for the sharp leading edge at $\alpha=0^{\circ}$. Cardone et al. [8] analyse the behaviour of a cylinder with two different blunt forebodies (sharpedged disk and hemispherical nose) at angles of attack from $0^{\circ}$ up to $20^{\circ}$ and find that, for the sharp-edged cylinder, the leading edge separation bubble, which is present for axisymmetric flow, still persists with increasing $\alpha$ but changes considerably in shape; instead, they do not notice any presence of a separation bubble for the round-nosed cylinder.

Carlomagno in a recent paper [9] analyses the behaviour of cylinders with three different diameters 12.7, 40 and $80 \mathrm{~mm}$ tested at different turbulence levels $\sigma_{u}$. He, in particular, finds that lowering the turbulence of the freestream makes the reattachment point to delay (e.g. from 1.2 for the cylinder with $D=80 \mathrm{~mm}$ at $\sigma_{u}=0.9 \%$ to 1.7 for $D=40 \mathrm{~mm}$ at $\sigma_{u}=0.1 \%$ or $1.8-2.0$ for $D=$ $12.7 \mathrm{~mm}$ at $\sigma_{u}=0.1 \%$ ). Differences between data relative to the two cylinders of $D=40$ and $80 \mathrm{~mm}$ have to be ascribed to the different turbulence level; instead, the most probable cause of differences between cylinders tested at the same value of $\sigma_{u}$ may be linked to the manufacture of the cylinder of $D=12.7 \mathrm{~mm}$. In fact, the external surface of the latter cylinder is heated starting at $0.3 D(0.1 D$ for $D=40 \mathrm{~mm})$ and the wall, at the leading edge, is welded to a $10 \mathrm{~mm} D$ copper bar that gives electrical continuity but acts as a heat sink. In addition, Carlomagno [9] finds separation on a round-nosed cylinder tested, at $0^{\circ}$ angle of attack, in a lower free stream $\sigma_{u}$.

In the present work, the behaviour of a cylinder with two different forebodies, sharp edge and round nose, placed at angle of attack in a low-turbulence stream, is investigated by means of infrared thermography. The aim is to assess the influence of the leading edge configuration at angles of attack.

\section{Experimental apparatus and technique}

The tests are performed in an open circuit wind tunnel having a $300 \times 400 \mathrm{~mm}^{2}$ rectangular test section which is $1.1 \mathrm{~m}$ long. The freestream turbulence intensity of the tunnel lies in the range $0.08-0.12 \%$ depending on the testing conditions.

The longitudinal cylinder has an outer diameter $D=40 \mathrm{~mm}$, an overall streamwise length of $300 \mathrm{~mm}$ with the lateral surface made of a printed circuit board (bonded to a fibreglass layer) to generate a constant Joule heat flux over it. The resulting pipe is very rigid and has an overall thickness of about $1.5 \mathrm{~mm}$. The copper conducting tracks of the printed circuit are $35 \mu \mathrm{m}$ thick, $3 \mathrm{~mm}$ wide, placed at $4 \mathrm{~mm}$ pitch and aligned perpendicularly to the cylinder axis. Two vertical brass struts, mated with mounting plates built in the upper and the lower wall of the tunnel, allow positioning the cylinder and varying its angle of attack with respect to the oncoming freestream. A sketch of the cylinder is shown in figure 1.

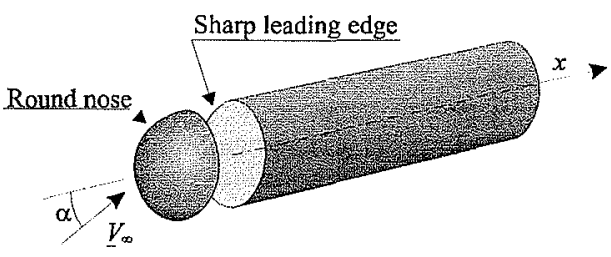

Fig. 1. Sketch of the cylinder forepart 


\section{http://dx.doi.org/10.21611/qirt.1996.028}

Two different configurations of the cylinder leading edge (nose) are tested: a sharp edge bluff nose and a hemispherical blunt one. The external surface of the cylinder, which is viewed by the infrared camera, is coated with a thin layer of black opaque paint, which has emissivity coefficient equal to about 0.95 in the wavelength of interest. The access window to the test section of the wind tunnel is simply made of bioriented polyetilene; calibration of the radiometer takes into account its presence.

The infrared thermographic system employed is the AGEMA Thermovision 900 . The infrared camera measures the temperature map of the model wall which is correlated to the heat transfer coefficient by means of the steady state heated-thin-foil technique [10]. In particular, for each pixel of the digitized thermal image, the convective heat transfer coefficient $h$ is calculated as:

$$
h=\left(\dot{q}_{w}-\dot{q}_{r}-\dot{q}_{c}\right) /\left(T_{w}-T_{\infty}\right)
$$

where $\dot{q}_{w}$ is the Joule heat flux, $\dot{q}_{r}$ are the losses due to radiative flux, $\dot{q}_{c}$ are the losses due to tangential conduction, $T_{w}$ and $T_{\infty}$ are the wall temperature and the free stream temperature, respectively. Because of the low value of the pertinent Biot number, the heated wall may be considered isothermal across its thickness.

The radiative thermal losses $\dot{q}_{r}$ may be evaluated according to the Stefan-Boltzmann law:

$$
\dot{q}_{r}=\varepsilon \sigma\left(T_{w}^{4}-T_{a}^{4}\right)
$$

where $\varepsilon$ is the total emissivity coefficient, $\sigma$ is the Stefan-Boltzman constant and $T_{a}$ is the ambient temperature. The thermal losses due to tangential conduction are neglected.

The heat transfer coefficients are computed in non-dimensional form by means of the local Nusselt number:

$$
N u=h D / \lambda
$$

where $D$ is the diameter of the cylinder and $\lambda$ the thermal conductivity coefficient of air evaluated at film temperature.

Tests are carried out for different Reynolds number Re defined in the conventional way:

$$
R e=V_{\infty} D / v
$$

where $V_{\infty}$ and $v$ are the free stream velocity in the tunnel test section, and the kinematic viscosity coefficient of air, respectively.

In order to measure temperatures in the whole heated zone and to account for the directional emissivity coefficient, three different thermal images in the azimuthal direction are taken and patched up.

\section{Results}

The results at zero angle of attack $\alpha=0^{\circ}$ show that, for the sharp leading edge, a maximum $N u$ is attained at $x / D=1.6-1.7$ regardless of the Reynolds number value. For the round nose, a maximum $N u$ is still found which is seen to move upstream from $x / D=0.7$ to $x / D=0.3$ as $R e$ increases from 26,000 to 89,000 . 
First of all, it must be remembered that the location of the maximum convective heat transfer does not exactly coincide with that of flow reattachment, which, as demonstrated by Sparrow et al. [4] for the sharp-edged cylinder, is located slightly downstream of this position. However, the position of the maximum $\mathrm{Nu}$ can be considered to determine the length of the separation bubble [8].

The present results confirm the findings of Carlomagno $[6,9]$ and support the contention that the freestream turbulence level is responsible for the formation of the leading edge separation bubble. The position of the reattachment point depends on the freestream turbulence level $\sigma_{u}$ in fact, for the sharp-edged cylinder the maximum $N u$ occurs at $x / D=1.6-1.7$ in both the present tests and those of Carlomagno $[6,9]$ which are carried out in a wind tunnel with $\sigma_{u}$ equal to about $0.1 \%$. Instead, other works $[3,4,8]$, which relate to higher values of $\sigma_{u}$, show a maximum $N u$ at $x / D=1.2-1.3$. Also for the round-nosed cylinder the length of the separation bubble is governed by the value of $\sigma_{u}$. The reattachment point, located at about $0.15 D$ in the tests of Peake and Tobak [7], appears delayed in the present tests, which, on the other hand, are in general agreement with Carlomagno [6,9]. On the contrary, results, presented by Cardone et al. [8], for a round-nosed cylinder, tested at a higher intensity turbulence level $(0.9 \%)$ of the freestream, show a monotonic decrease of the Nusselt number with increasing the longitudinal coordinate. This may be due to the shorter length of the separation bubble, which is probably positioned in the initial zone $(0.25 D)$ of the leading edge not included in the measurements of Cardone et al. [8].

Data are presented as $\mathrm{Nu}$ isocontours over the cylinder surface for the two leading edge configurations, for $\operatorname{Re}=71,000$ and $\alpha=0^{\circ}$ in figures $2 \mathrm{a}$ and $2 \mathrm{~b}$. It has to be pointed out that, due to the end-conduction effects near the forebody, the portion of the cylinder for which the infrared camera gives reducible data actually starts at $x / D=0.2$ and ends at $x / D=5$. No wortwhile heat transfer effects, or flow evolution, seem to appear after $5 D$.
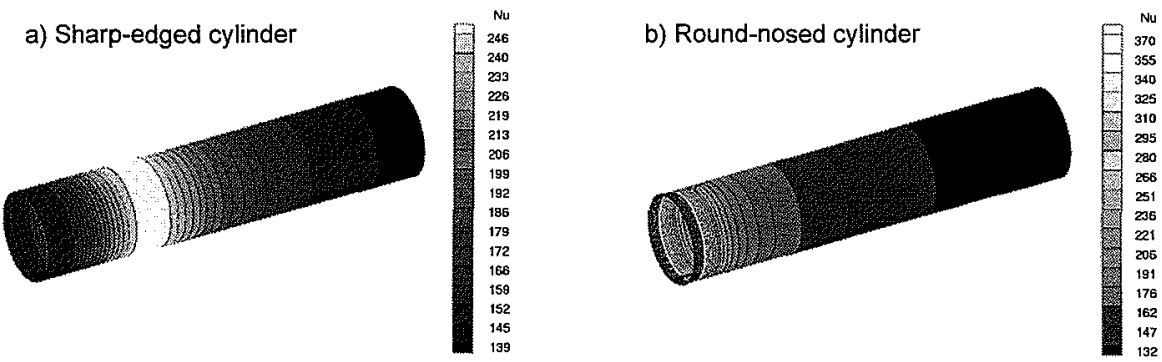

Fig. 2. Nu isocontours for $\operatorname{Re}=71,000$ and $\alpha=0^{\circ}$

For the round-nosed cylinder at $\alpha=0^{\circ}$ (figure $2 \mathrm{~b}$ ), the maximum $\mathrm{Nu}$ is positioned slightly downstream of the junction of the hemispherical nose to the cylindrical body $(x / D=0.35$ ), extends for a very narrow zone and attains there values greater than those relative to the sharp-edged cylinder (figure 2a); however, the Nusselt number shows firstly a sharp decrease (e.g. a reduction of about $50 \%$ of the maximum is evident at $x / D=1.5$ ) and after reaches practically the same value relative to the sharp leading edge towards $x / D=5$.

The heat transfer pattern changes considerably as the angle of attack is increased from $0^{\circ}$ to $10^{\circ}$ and, for both configurations (sharp and round), the maximum $\mathrm{Nu}$ moves upstream on the windward side while it remains quite in the same position on the leeward one. In the tested range the Reynolds number does not affect the position of the maximum $\mathrm{Nu}$ for the case of a sharp leading edge; instead, in the case of a round nose, the maximum $\mathrm{Nu}$ moves upstream as $R e$ 
increases. In addition, for the round-nosed cylinder, the maximum $\mathrm{Nu}$ tends to disappear on the windward side as $\alpha$ increases and this is in general agreement with the finding of Peake and Tobak [7]. It is worth noting that on the windward side for $\alpha=5^{\circ}$ the maximum $\mathrm{Nu}$ is present for $R e=89,000$, becomes much milder as $R e$ is decreased and disappears for $\operatorname{Re}=26,000$; otherwise, for $\alpha=10^{\circ}$ no maximum $N u$ is found for all the tested Reynolds number values.

The $\mathrm{Nu}$ isocontours for $\operatorname{Re}=71,000$ and $\alpha=5^{\circ}$ and $10^{\circ}$ are presented in the pictures of figures 3a-3d. As can be seen, zones of maximum Nusselt number are present on the windward side of the cylinder with sharp leading edge (figures 3a and 3c) and on the leeward side of the cylinder with hemispherical nose (figures $3 b$ and $3 d$ ).

It has to be pointed out that the leading edge separation bubble moves upstream as the angle of attack increases and changes considerably in shape due to a substantial deçrease of its length on the windward side; this entails variation in the location of the reattachment point and so on the distribution of the heat transfer. For the sharp leading edge at $\alpha=0^{\circ}$ a maximum $\mathrm{Nu}$ occurs at about 1.6D. As the angle of attack is increased the intensity of the heat transfer varies azimuthally with a significant increase on the windward side where the maximum $N u$ is attained at about $0.7 D$ for $\alpha=10^{\circ}$ (figure 3c). In the case of the cylinder with hemispherical nose at $\alpha=0^{\circ}$ the maximum $\mathrm{Nu}$ is attained at a distance of about $0.35 \mathrm{D}$ for $R e=71,000$. The separation bubble, in the latter case, is considerable shorter already at $\alpha=0^{\circ}$ and further shortens as the angle of attack increases with a maximum $N u$ probably located at $x / D<0.2$ for $\alpha=5^{\circ}$ which is not included in measurements and so not visible in figure $3 \mathrm{~b}$. On the other hand, if the angle of attack is further increased $\left(\alpha=10^{\circ}\right)$ the bubble disappears on the windward side giving rise to the formation of two vortices which can be assumed to coincide with the saddle points observed by Peake and Tobak [7] on either side of the nodal separation point on the leeward side. This justifies the presence of the two thermal reattachment points on the leeward side in figure $3 \mathrm{~d}$.

\section{Conclusions}

Measurements of convective heat transfer coefficients, over the surface of a cylinder longitudinal to a free-stream, have been made by means of infrared thermography applied to the heated-thin-foil technique. The presence of a leading edge thermal separation bubble is recognised its length being determined by the position of the maximum heat transfer coefficient. This bubble is present also in the case of a round-nosed cylinder, but its length is shorter as compared to that relative to the sharp leading edge. As the angle of attack is increased the bubble shape changes with a substantial decrease of its length on the windward side entailing azimuthal variation of the heat transfer intensity. A comparison of the present data with literature shows a strong influence of the freestream turbulence level on the length of the separation bubble and so on the position of the reattachment point; the lower the turbulence level of the flow the more delayed the reattachment.

\section{REFERENCES}

[1] CHANG (P.K.). - Separation of flow, Pergamon Press, 1966.

[2] OTA (T.). - An axisymmetric separated and reattached flow on a longitudinal blunt circular cylinder, J. Applied Mechanics, Vol. 42, pp. 311-315, 1975.

[3] OTA (T.) and KON (N.). - Heat transfer in an axisymmetric separated and reattached flow over a longitudinal blunt circular cylinder, J. Heat Transfer, Vol. 99, pp. 155-157, 1977.

[4] SPARROW (E.M.), KANG (S.S.) and CHUCK (W.). - Relation between the points of flow reattachment and maximum heat transfer for regions of flow separation, Int. J. Heat Mass Transfer, Vol. 30, pp. 1237-1246, 1987. 
[5] KIVA (M.), MOCHIZUKI (O.), TAMURA (H.), NOZAWA (T.), ISHIKAWA (R.) and KUSHIOKA (K.). - Turbulence properties of an axisymmetric separation-and-reattaching flow, AIAA J. Vol. 29, pp. 936-941, 1991.

[6] CARLOMAGNO (G.M.). - Heat transfer measurements and flow visualization performed by means of infrared thermography, Proc. Eurotherm Sem. $\mathrm{N}^{\circ} 46$, Heat Transfer in Single Phase Flows 4, Di Marco P. Ed., pp. 45-52, Pisa, 1995.

[7] PEAKE (D. J.) and TOBAK (M.). - Three-dimensional flows about simple components at angle of attack, AGARD-LS-121, paper 2, 1982.

[8] CARDONE (G.), BURESTI (G.) and CARLOMAGNO (G.M.). Heat transfer to air from a yawed circular cylinder, to be published on Atlas of Visualization III, 1996.

[9] CARLOMAGNO (G:M.). - Quantitative infrared thermography in heat and fluid flow, in Optical Methods and Data Processing in Heat and Fluid Flow, IMechE Conf. Trans. 1966-3, 279-290, London 1996.

[10]CARLOMAGNO (G.M.), DE LUCA (L.). - Infrared Thermography in Heat Transfer, in Handbook of Flow Visualization, Yang W.J. ed., pp. 551-553, 1989. 